\title{
Recorrido geológico y minero desde Híjar a Samper de Calanda y a Castelnou, a través del patrimonio geológico y minero de la comarca del Bajo Martín
}

Josep Maria Mata-Perelló

Andrés Pocoví Juan

Joaquim Sanz Balagué

Jaume Vilaltella

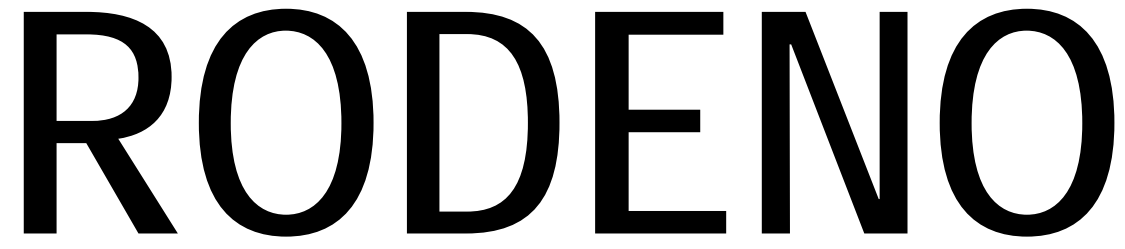

\section{REVISTA DE GEOLOGÍA ARAGONESA}

\section{n. 4}

DIECIEMBRE 2015

Como citar el artículo: Mata Perelló, Josep M. [et al.] Recorrido geológico y minero desde Híjar a Samper de Calanda y a Castelnou, a través del patrimonio geológico y minero de la comarca del Bajo Martín. En: Rodeno [revista electrónica]: revista de geología aragonesa. Manresa: Museu de Geologia Valentí Massachs, DICIEMBRE 2015. núm. 4. ISSNe: 1131-5393 [Data de consulta] Disponible en: http:// ... 


\title{
RECORRIDO GEOLÓGICO Y MINERO DESDE HÍJAR A SAMPER DE CALANDA Y A CASTELNOU, A TRAVÉS DEL PATRIMONIO GEOLÓGICO Y MINERO DE LA COMARCA DEL BAJO MARTÍN
}

\author{
Josep Maria Mata-Perelló \\ Museu de geologia Valentí Masachs, Escola Politècnica Superior d'Enginyeria de Manresa \\ (EPSEM), Universitat Politècnica de Catalunya · BarcelonaTech (UPC), 08272 Manresa, Spain
}

\author{
Andrés Pocovi Juan \\ Universidad de Zaragoza, Departamento de Ciencias de la Tierra ( DCT- UZ) \\ Joaquim Sanz Balagué \\ Departament d'Enginyeria Minera i Recursos Naturals (EMRN), Escola Politècnica Superior \\ d'Enginyeria de Manresa (EPSEM), Universitat Politècnica de Catalunya - BarcelonaTech \\ (UPC), 08272 Manresa, Spain
}

\section{Jaume Vilaltella Farràs.}

Sociedad Española para la Defensa del Patrimonio Geológico y Minero (SEDPGYM)

Palabras clave: patrimonio minero; patrimonio geológico; Zaragoza

\section{Resumen}

El recorrido de este itinerario, se desarrollará íntegramente por una sola unidad geológica, por la Depresión Geológica del Ebro (en donde se iniciará y en donde finalizara el recorrido).

Así, el recorrido del presente itinerario se iniciará en las inmediaciones de Hijar, dentro de la unidad geológica antes mencionada, para finalizar en las cercanías de Castelnou, yendo hacia Escatrón; en ambos casos (al igual que todo el recorrido), dentro de la Depresión Geológica del Ebro

Así, en este recorrido, se irán encontrando afloramientos de los materiales cenozoicos, en su mayoría de carácter detrítico, pertenecientes generalmente al Oligoceno. No obstante, cabe señalar los importantes afloramientos de yesos y arcillas oligocénicos. 


\section{ADVERTENCIAS PREVIAS}

Como en otros recorridos de RECONOCIMIENTO GEOLÓGICO (o de RECONOCIMIENTO GEOLÓGICO Y MINERO), el recorrido se compondrá de diversas PARADAS. En este caso serán seis.

Por otra parte, habrá que tener en cuenta, en todo momento, especialmente antes de empezar los recorridos de los diferentes tramos, el estado de los caminos y carreteras, por donde transitará el recorrido.

Precisamente, en este itinerario, se discurrirá en una pequeña parte del recorrido por caminos de tierra. Al respecto, cabe decir que prácticamente todo este tramo se halla en buenas condiciones.

Finalmente, como ya hacemos en otros recorridos similares, queremos decir que hace falta tener un cuidado muy especial en el respeto a la naturaleza, a lo largo de todo el recorrido del itinerario, y también fuera de él.

\section{BREVE INTRODUCCIÓN GEOGRÁFICA}

El recorrido del presente itinerario se efectuará exclusivamente por una comarca aragonesa, por la del Bajo Martín. Así, transitara siguiendo la ruta entre Hijar, Samper de Calanda, Jaciel y Castelnou siguiendo el valle del río Martín, hasta llegar al municipio de Escatrón (perteneciente a la vecina comarca de la Ribera Baja del Ebro, de la provincia de Zaragoza).

\section{OBJETIVOS GENERALES DE ESTE ITINERARIO}

En este itinerario, los objetivos generales que se han de conseguir, se pueden concretar en los siguientes aspectos:

1.- Estudio y reconocimiento de los materiales cenozoicos (en buena parte del Oligoceno) situados en la Depresión Geológica del Ebro, que iremos encontrando a lo largo del recorrido del itinerario, entre las localidades de Hijar, Samper de Calanda, Jaciel y Castelnou.

2.- Visión de algunas de las antiguas explotaciones encontradas a lo largo del recorrido del itinerario

3.- Visión de los diferentes lugares directamente relacionados con el Patrimonio Geológico y Minero que iremos encontrando a lo largo del recorrido de este itinerario. 


\section{ANTECEDENTES BIBLIOGRÁFICOS}

En relación con este itinerario, no conocemos ningún antecedente, relativo a otro itinerario que discurra por este lugar. En este sentido, este itinerario constituye un antecedente, si no estamos equivocados.

Por otra parte, haremos mención de algunos trabajos, de carácter geológico generalista, que corresponden a los trabajos del IGME (1972, 1974 y 1975), relativos al Mapa Geológico de España (a Escala 1.200.000), al Mapa Metalogenético de España y al Mapa de Rocas Industriales de España. Igualmente, nos referiremos a los trabajos del IGME (1980 y 1981), referidos al Mapa Geológico de España a escala 1:50.000.

Con respecto a las mineralizaciones que iremos encontrando, mencionaremos los trabajos de: CALVO et altri (1988); MAESTRE (1845); así como nuestros trabajos: MATA-PERELLÓ (1987 y 1994).

También mencionaremos el trabajo de PRAMES (2005) dedicado a la comarca del Bajo Martín. Así como el del GOBIERNO DE ARAGÓN (2001), dedicado a los Puntos de Interés Geológico de Aragón.

Finalmente, diremos que todos estos trabajos (así como otros que ahora no hemos aludido), figurarán mencionados, por orden alfabético, en el apartado dedicado a las REFERENCIAS BIBLIOGRÁFICAS.

\section{RECORRIDO DEL ITINERARIO}

Este recorrido se iniciará en las inmediaciones del pueblo de Hijar (la capital comarcal), en donde se realizaran dos paradas. Seguidamente, el recorrido se dirigirá hacía el pueblo vecino de Samper de Calanda, por se realizaran dos nuevas paradas.

Tras ello, el recorrido se dirigirá hacía los municipios de Jaciel y Castelnou, en donde se efectuaran las últimas paradas. Luego, el recorrido continuará hasta el límite comarcal, en donde finalizará, muy cerca de Escatrón.

\section{DESCRIPCIÓN DEL ITINERARIO}

Como de costumbre, haremos una serio de PARADAS (o ESTACIONES), en donde se realizaran diversas explicaciones en torno a las características del lugar en donde se halla la PARADA. 
Por otra parte, en ellas haremos mención del término municipal dónde se encuentran, así como del número del "Mapa Topográfico Nacional (a escala 1:50.000, que indicaremos entre paréntesis. Así, ahora (en este recorrido) utilizaremos solamente una hoja: concretamente la $\mathbf{4 4 1}$ (o de Hijar).

Así, la relación ordenada de las paradas que constituyen el recorrido de este itinerario, es la siguiente:

\section{PARADA 1 - CONDICIONAL. FÁBRICA DE CERÁMICA DE HÍJAR, (término municipal de Hijar, comarca del Bajo Martín). (Hoja 441).}

El recorrido del presente itinerario lo habremos iniciado en la localidad de Hijar, y podemos realizar la primera parada en la antigua cerámica. Ésta se halla al inicio de la carretera que conduce a Albalate del Arzobispo, tras cruzar la carretera nacional que une Alcañiz con Zaragoza, partiendo de Hijar. En este lugar es en donde hemos efectuado la última parada del primer itinerario.

Este lugar, a igual que la población de Hijar, se halla totalmente situado en la Depresión Geológica del Ebro, entre afloramientos de los materiales cenozoicos del Oligoceno. Estos materiales (las lutitas y calcolutitas ocres) han sido explotados en este lugar, con la finalidad de ser utilizados como materia prima para la fábrica de cerámica.

En lo que concierne a esta. cabe decir que se halla en un avanzado estado de deterioro. En todos los aspectos. 


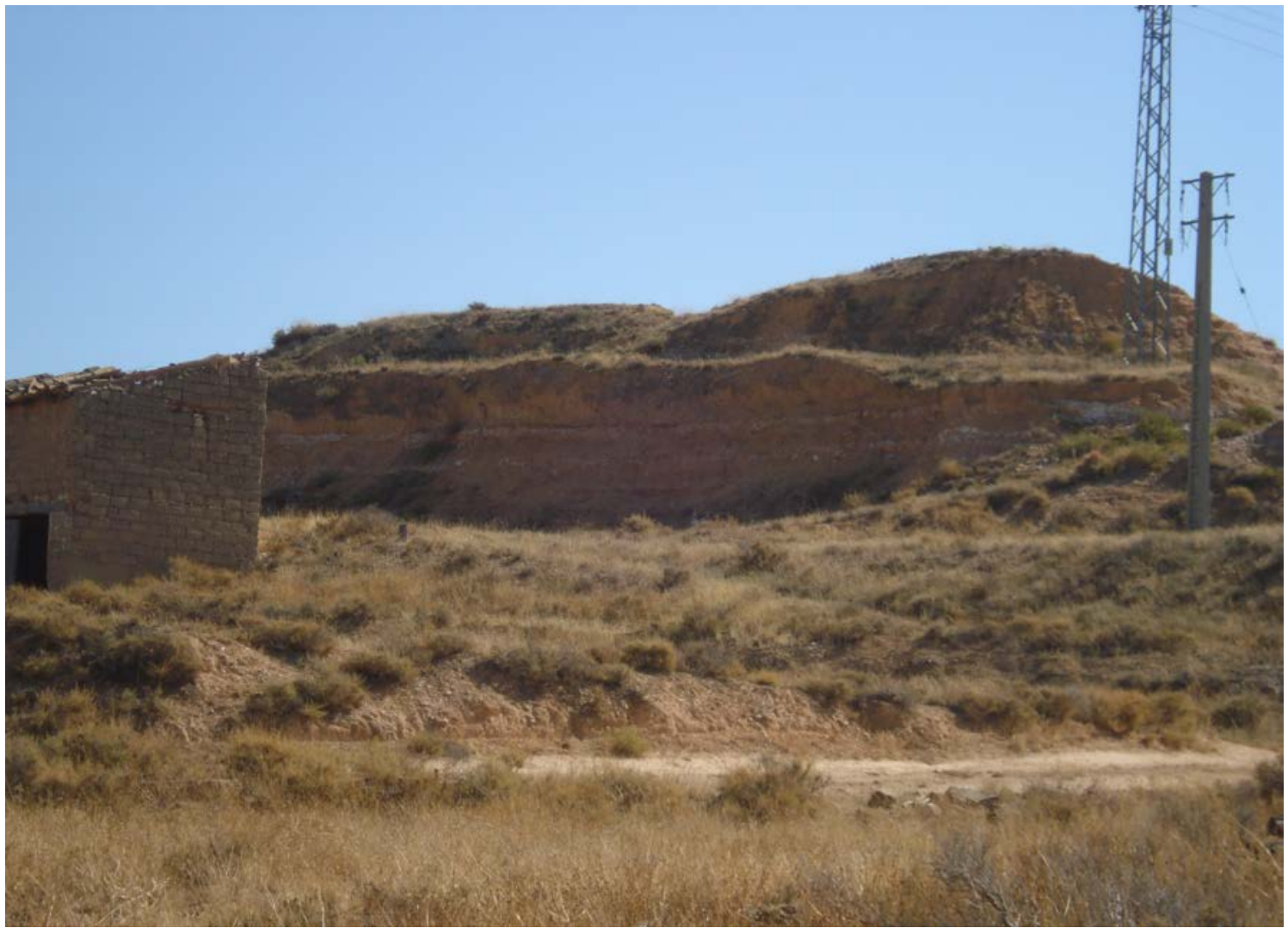

Antigua explotación de los materiales lutíticos 


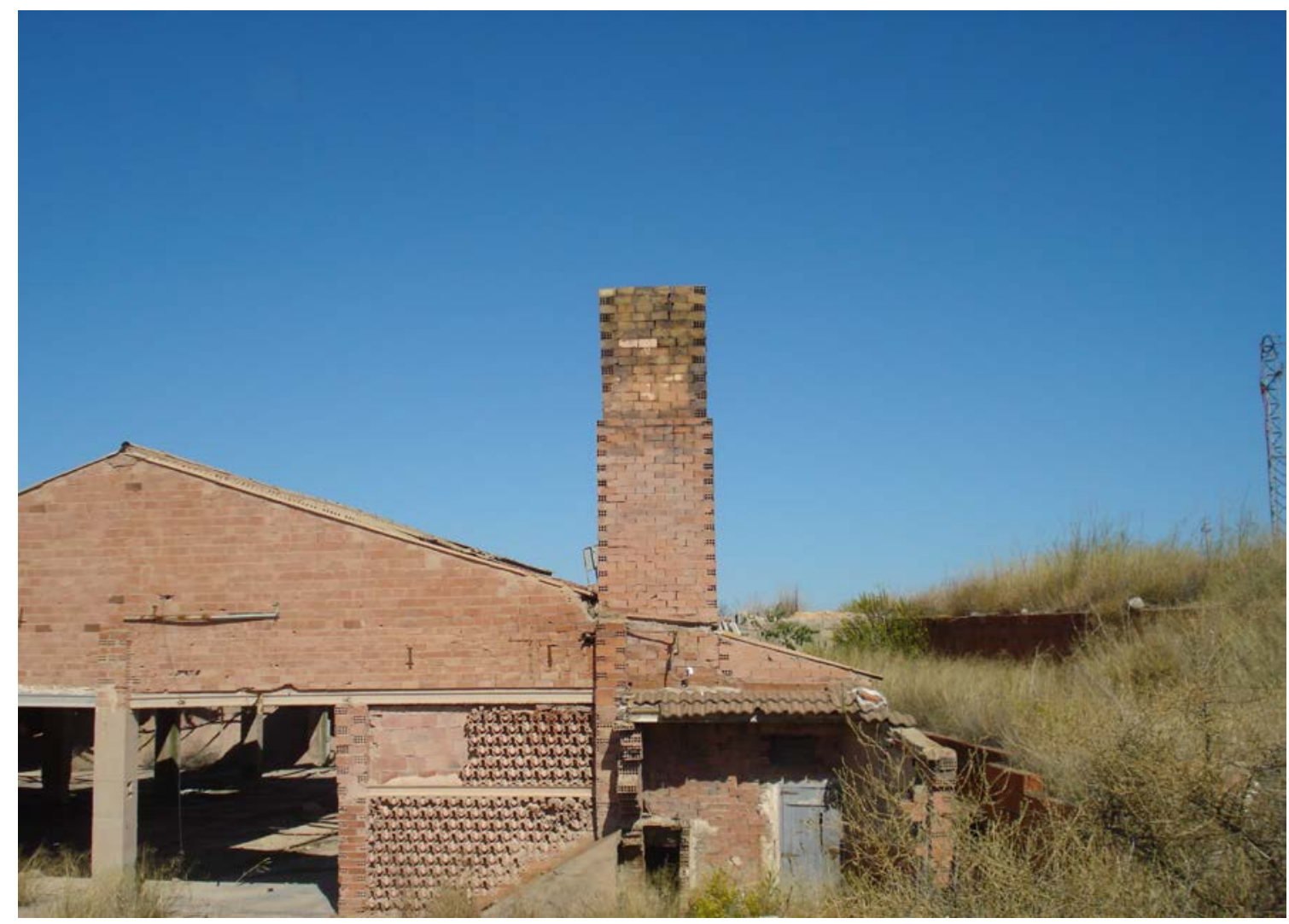

Restos de la fábrica de cerámica

PARADA 2. TERRERAS DE LA CARRETERA A SAMPER DE CALANDA, (término municipal de Hijar, comarca del Bajo Martín). (Hoja 441).

Tras realizar la parada anterior, conviene de nuevo ir hacía Hijar, con la intención de tomar la carretera que se dirige hacía el cercano pueblo de Samper de Calanda. Sin embargo, a la salida del primer pueblo, convendrá hacer una nueva parada. Así, desde la parada anterior, habremos recorrido unos $3 \mathrm{Km}$.

En este recorrido, habremos estado situados, en todo momento, entre afloramientos de los materiales cenozoicos del Oligoceno. Estos materiales rellenan la Depresión Geológica del Ebro, en donde estamos ahora situados.

Rn este lugar hay un afloramiento de los materiales lutíticos y calcolutíticos, que han sido explotados. 


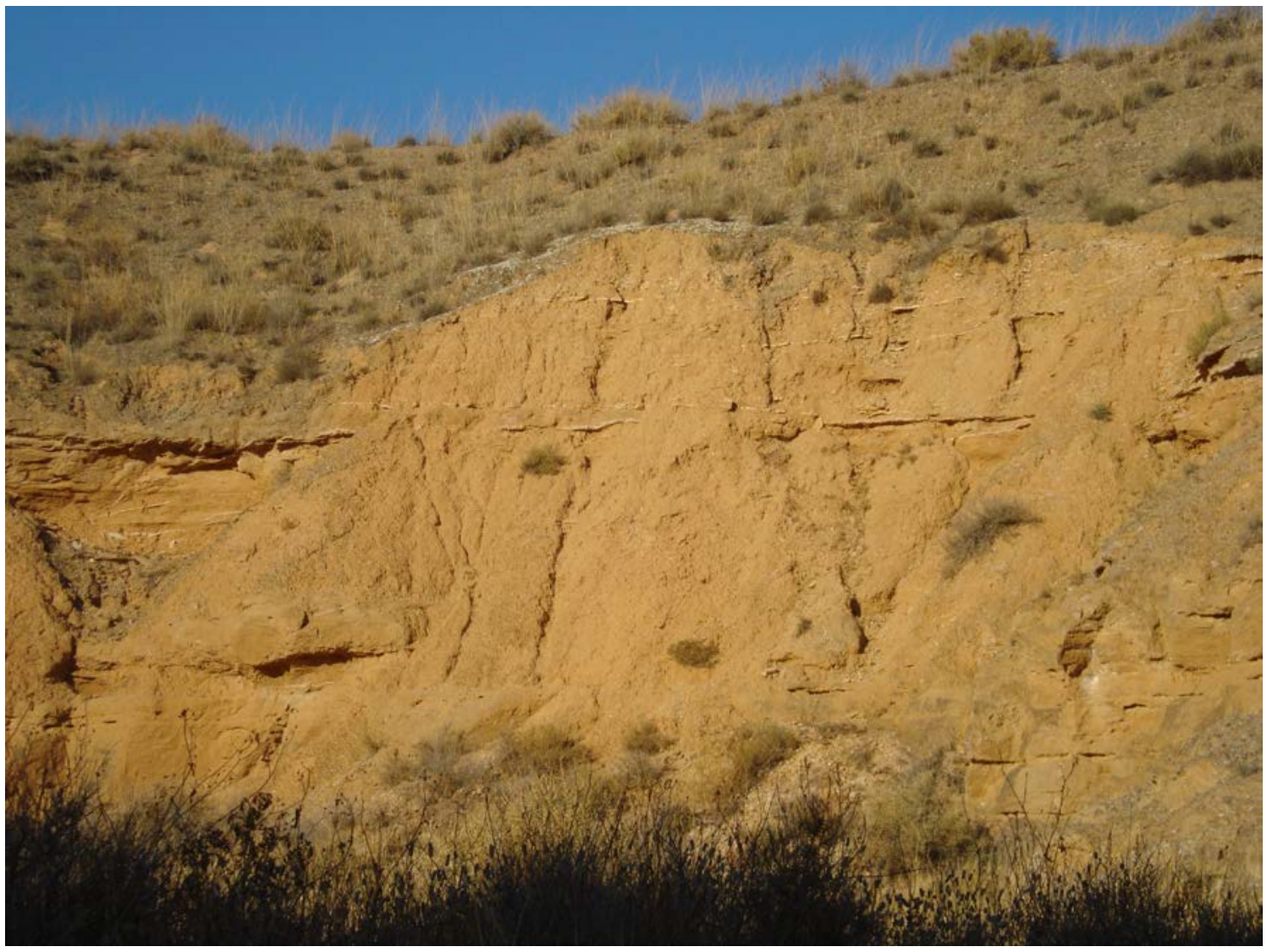

Pequeña explotación de calcolutitas. Hijar, carretera a Samper de Calanda

\section{PARADA 3. GRAVERA DE LA HUERTA, (término municipal de Samper de Calanda, comarca del Bajo Martín). (Hoja 441).}

Después de efectuar la parada anterior, cabe continuar por la carretera comarcal que se dirige hacía el cercano pueblo de Samper de Calanda. Poco antes de llegar a él, a medio camino entre los dos pueblos, nos convendrá tomar un camino que sale por la izquierda de la carretera. Este camino nos lleva a una antigua gravera. Así, desde la parada anterior habremos recorrido unos $2 \mathrm{Km}$ más.

En este recorrido habremos continuado encontrando afloramientos de los materiales cenozoicos ya citados en la parada anterior. A menudo se hallan recubiertos por materiales cuaternarios, como en el lugar de la parada. Aquí, aparecen unos niveles de gravas pertenecientes a la terraza media del río Martín. Cabe decir que fueron explotados en una antigua gravera. 


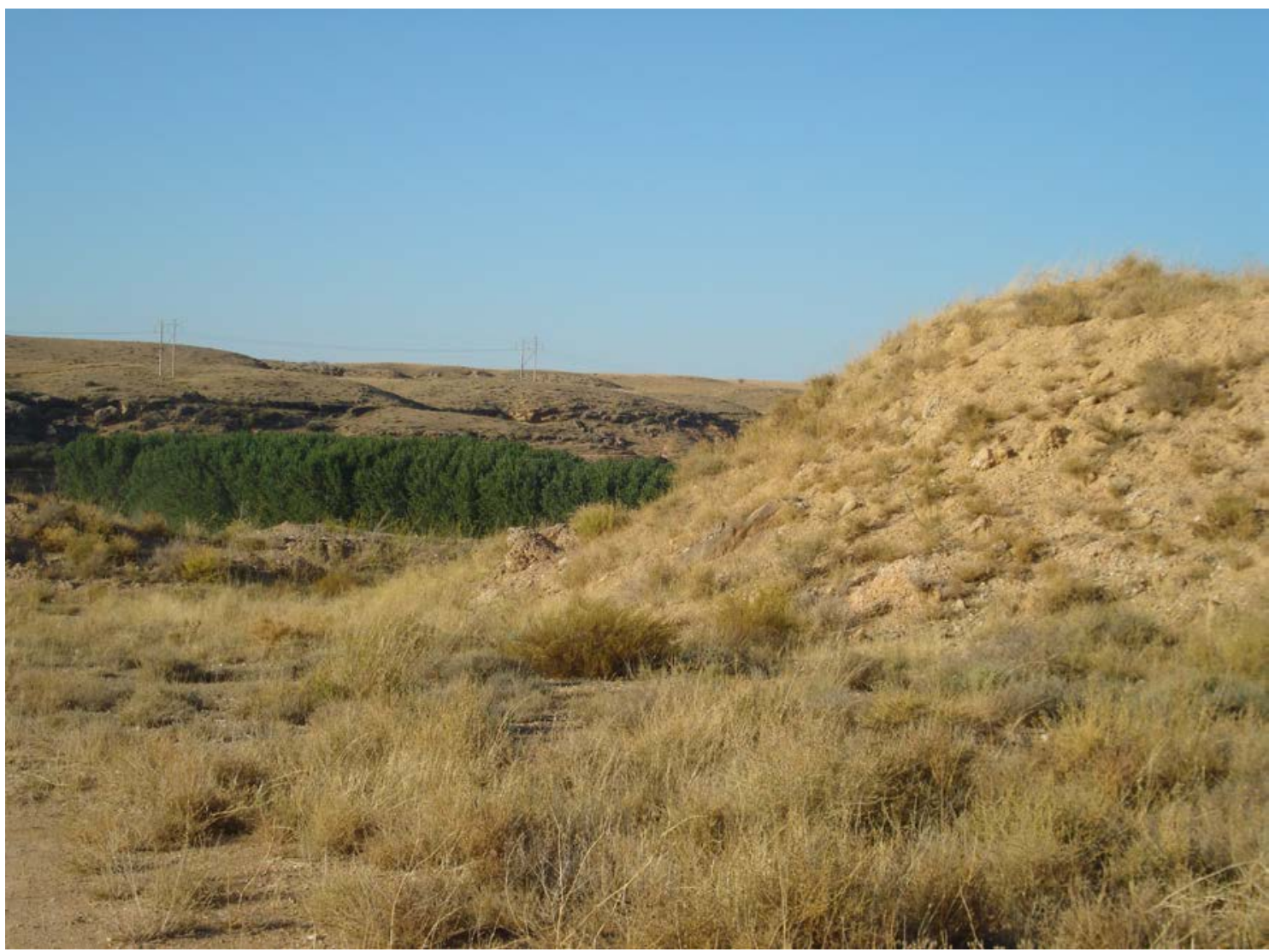

Restos de la gravera de Samper de Calanda

PARADA 4 - CONDICIONAL. CAMINO DE VALDELIEGO, (término municipal de Samper de Calanda, comarca del Bajo Martín). (Hoja 441).

Después de realizar la parada anterior, conviene seguir hacía el pueblo de Samper de Calanda. Luego, será menester ir hacía la estación del ferrocarril. Luego, convendrá tomar el camino de Valdeliego, en donde realizaremos una nueva parada, a unos $3 \mathrm{Km}$ de la anterior.

En este recorrido, hemos continuado encontrando afloramientos de los materiales cenozoicos y cuaternarios. Como en los casos anteriores, estos materiales se hallan en la Depresión Geológica del Ebro, en donde ahora estamos. Finalmente, cabe decir que aquí ha habido una pequeña explotación de los niveles lutíticos y calcolutíticos oligocénicos.

PARADA 5 - CONDICIONAL CANTERA DE ALABASTRO DE VAL DE $R E N$, (término municipal de Jaciel, comarca del Bajo Martín). (Hoja 441).

Después de efectuar la parada anterior, conviene regresar a Samper de Calanda, para ir luego hacía Jaciel. Luego, desde ahí iremos hacía el Val del Ren. Ahí 
realizaremos una nueva parada, en una de las explotaciones pertenecientes al municipio de Jaiciel. Así, desde la parada anterior, habremos recorrido unos $3 \mathrm{Km}$ más.

En este recorrido, hemos encontrado importantes afloramientos de yesos oligocénicos. Estos materiales han sido explotados en diversos lugares, como en el de la presente parada. Así, aquí se han explotado los niveles de yesos, para ser utilizados como alabastro.

PARADA 6. CANTERA DE ALABASTRO DE VAL DE REN, (término municipal de Castelnou, comarca del Bajo Martín). (Hoja 441).

Después de efectuar la parada anterior, cabe hacer un pequeño recorrido (de menos de 0’5 Km), con la intención de llegar hasta otra cantera similar a la anterior, situada ahora en el municipio de Castelnou.

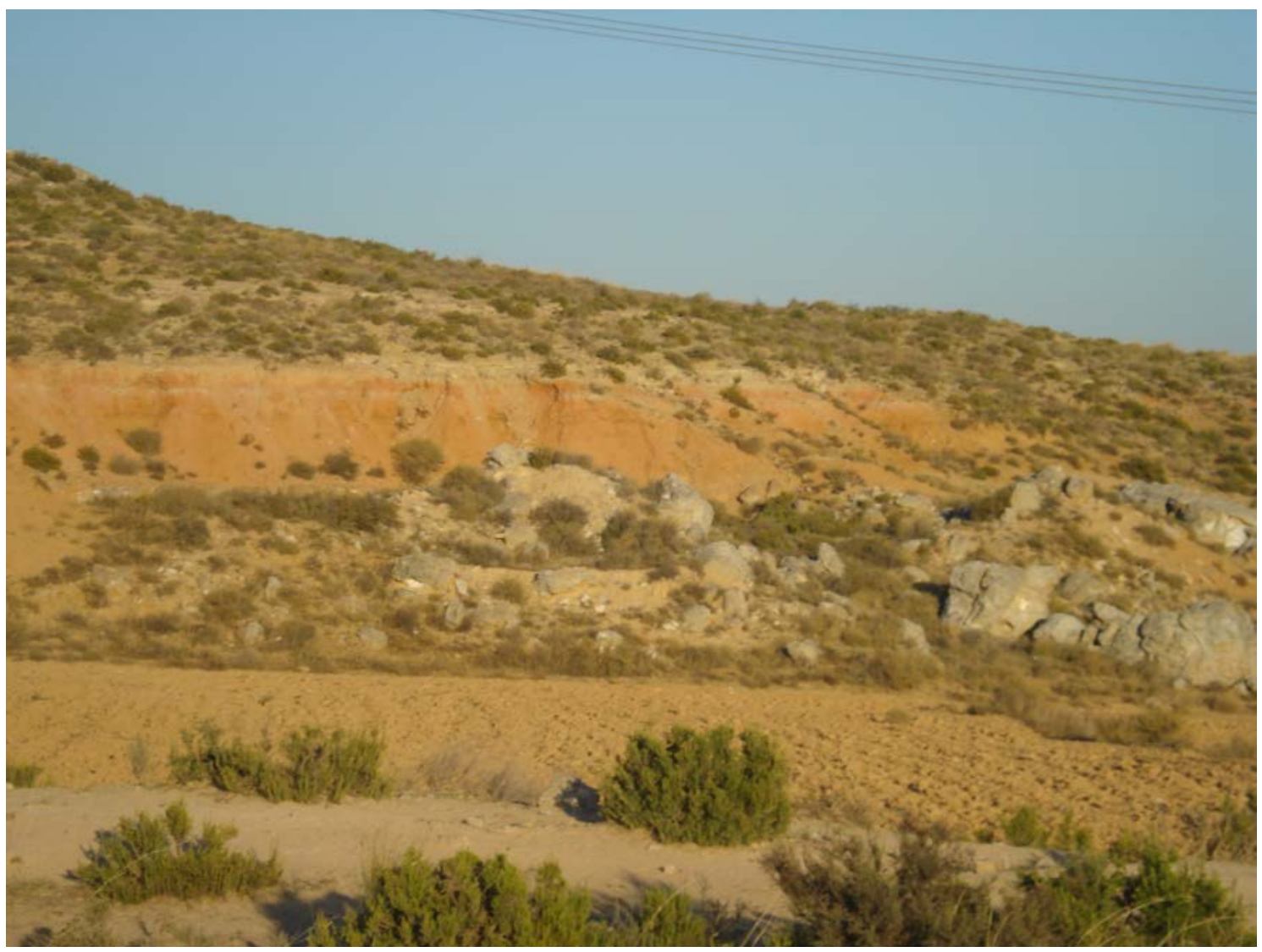

Restos de la explotación de alabastro del Valle del Ren (Castelnou)

Así, en este lugar se han explotado los niveles de yeso (concretamente de alabastro) que se hallan intercalados entre tramos de calcolutitas ocres oligocénicas. 
Cabe decir que por este sector existen numerosas explotaciones de alabastro, aunque todas ellas están abandonadas en la actualidad.

\section{EN ESTE LUGAR FINALIZA EL ITINERARIO}

\section{BIBLIOGRAFÍA}

CALVO, M. et altri (1988). - Minerales de Aragón, Colección Temas Geológicos, 207 Pág. Zaragoza

GOBIERNO DE ARAGÓN (2001).- Puntos de Interés Geológico de Aragón. Consejería de Medio Ambiente del Gobierno de Aragón. Zaragoza

IGME (1972).- Mapa Geológico de España a escala 1:200.000 (Síntesis de la cartografía existente). Hoja y Memoria na 32 (Zaragoza). Inst. Geol. Min. España

IGME (1973).- Mapa Metalogenético de España a escala 1:200.000. Hoja y Memoria ${ }^{a}$. 32 (Zaragoza). Inst. Geol. Min. España

IGME (1975).- Mapa de Rocas Industriales de España a escala 1:200.000. Hoja y Memoria $n^{a}$. 32 (Zaragoza). Inst. Geol. Min. España

IGME (1980-1981).- Mapa Geológico de España a escala 1:50.000 (2a Serie). Hojas y Memorias ns. 441 (Hijar). Inst. Geol. Min. España

MAESTRE, A. (1845).- Descripción geognóstica del Distrito Minero de Cataluña y Aragón. Anales de Minas, t. III. Madrid

MATA - PERELLÓ, J.; (1987).- Introducción al conocimiento de las mineralizaciones aragonesas. Mineralogistes de Catalunya, t.III, pp. 258-265. Barcelona

MATA - PERELLÓ, J.M. (1994).- Inventario Mineralógico de la comarca de la Tierra Baja - Martín Rodeno, 28. 21 Pág. Manresa

PRAMES (2005).- Bajo Martín. Colección RUTASCAL por Aragón. Prames, Gobierno de Aragón. 119 pag. Zaragoza. 